\title{
Optimum Weights to Which Pigs Should Be Carried in Puerto Rico for Maximum Profits
}

\author{
Ismael Carlo ${ }^{1}$
}

\section{INTRODUCTION}

The importance of the swine industry in Puerto Rico has been increasing rapidly in the past few years. New and bigger swine herds have been established in the Island and several farmers are substituting garbage for concentrate feeding. A few farmers are already buying the ingredients and mixing their own rations on their farms. Tables of optimum weight to which pigs should be carried for maximum profit should be valuable as a guide for all swine producers in the Island.

Although tables of this kind were prepared for Durocs and Native pigs in $1949(1)^{2}$, it was felt that, with more data available, their revision was necessary. At the same time a table was needed for the crossbred pigs developed at this Station.

\section{METHODS}

The tables presented herein were prepared on the basis of data collected from 18 Duroc Jersey barrows and 73 Landrace Large Black Duroc crossbred barrows and are given separately.

The pigs considered in this study entered feeding trials which extended from weaning, at 56 to 182 days of age. The feed consumed and the gain in weight of the pigs was recorded every 14 days. The data were obtained from eight feeding trials covering the period from 1951 to 1957.

The feed used in all these trials consisted of concentrated rations mixed at the experimental farm at Lajas Substation with approximately 15.0percent crude-protein content, 3.0 percent of fat, and 6.5 of fiber.

The data collected were plotted as growth curves in a graph. The maximum weights for optimum profit were calculated by the method of González Chapel and Cabrera $(1)^{2}$. This was based on the liveweight of hogs selling at from 15 to 31 cents a pound, with feed costs varying from $\$ 3.00$

1 Assistant Animal Husbandman, Agricultural Experiment Station, University of Puerto Rico, Río Piedras, P. R. The author wishes to express his appreciation to Assistant Director Antonio González Chapel of the Agricultural Experiment Station, under whose leadership most of these experiments were conducted, for his assistance in the preparation of this paper.

2 Italic numbers in parentheses refer to Literature Cited, p. 37. 
TABLE 1.-Optimum weights for maximum profits in Duroc Jersey barrows at per pound selling price of hogs (liveweight) indicated ${ }^{1}$

\begin{tabular}{|c|c|c|c|c|c|c|c|c|c|c|c|c|c|c|c|c|c|c|}
\hline \multirow{2}{*}{$\begin{array}{c}\text { Feed } \\
\text { cost per } \\
100 \mathrm{lb} .\end{array}$} & \multicolumn{2}{|c|}{$\$ 0.15$} & \multicolumn{2}{|c|}{ \$0.17 } & \multicolumn{2}{|c|}{ \$0.19 } & \multicolumn{2}{|c|}{$\$ 0.21$} & \multicolumn{2}{|c|}{$\$ 0.23$} & \multicolumn{2}{|c|}{ s0.25 } & \multicolumn{2}{|c|}{ \$0.27 } & \multicolumn{2}{|c|}{$\$ 0.29$} & \multicolumn{2}{|c|}{ so.31 } \\
\hline & F & W & $\mathrm{F}$ & $\mathbf{w}$ & $\mathbf{F}$ & $\mathbf{W}$ & $\mathbf{F}$ & $w$ & $\mathbf{F}$ & $\mathbf{W}$ & $\mathbf{F}$ & $\mathbf{w}$ & F & $w$ & $\mathbf{F}$ & w & F & w \\
\hline 3.00 & 380 & 123 & 519 & 149 & 642 & 170 & 753 & 186 & 854 & $200 \mid$ & 946 & 212 & 1,031 & 222 & 1,111 & 230 & 1,184 & 238 \\
\hline 3.25 & 291 & 105 & 430 & 133 & 553 & 155 & 664 & 173 & 790 & 192 & 857 & 201 & 943 & 211 & 1,022 & 221 & 1,096 & $\mid 229$ \\
\hline 3.50 & 209 & 86 & 348 & 117 & 471 & 14] & 582 & 160 & 683 & 176 & 775 & 190 & 861 & 201 & 940 & 211 & 1,013 & $\mid 220$ \\
\hline 3.75 & 132 & 68 & 271 & 100 & 395 & 126 & 505 & 147 & 606 & 164 & 699 & 179 & 784 & 191 & 863 & 202 & 937 & 211 \\
\hline 4.00 & 61 & 49 & 200 & 84 & 323 & 111 & 459 & 138 & 535 & 152 & 627 & 168 & 712 & 181 & 792 & 192 & 866 & 202 \\
\hline 4.25 & - & - & 132 & 68 & 256 & 97 & 367 & 116 & 467 & $\mid 140$ & $560 \mid$ & 156 & 645 & 170 & 724 & 182 & 798 & 193 \\
\hline 4.50 & - & - & 69 & 51 & 192 & 82 & 303 & $\mid 107$ & 404 & $|128|$ & $497 \mid$ & 145 & 582 & 160 & 661 & 173 & 735 & 184 \\
\hline 4.75 & - & - & 9 & 35 & 132 & 68 & 243 & 94 & 344 & $\mid 116$ & 437 & 134 & 522 & 150 & 601 & $|163|$ & 675 & 175 \\
\hline 5.00 & - & - & - & - & 76 & 53 & 187 & 81 & 287 & 104 & 380 & 123 & 465 & 140 & 544 & 154 & 618 & 166 \\
\hline 5.25 & - & - & - & - & 21 & 39 & 132 & 68 & 233 & 92 & 326 & 112 & 411 & 129 & 490 & 144 & 564 & 157 \\
\hline 5.50 & - & - & - & - & - & - & 81 & 54 & 182 & $|80|$ & $|274|$ & 101 & 360 & $\mid 119$ & 439 & 134 & 513 & 148 \\
\hline
\end{tabular}

${ }^{1} \mathrm{~F}=$ feed consumed to reach optimum weight; $W=$ weight of the animal for maximum profit. Pigs should not be raised under conditions designated by the use of dashes.

TABLE 2.-Optimum weights for maximum profits in Landrace Duroc barrows at per pound selling price of hogs (liveweight) indicated"

\begin{tabular}{|c|c|c|c|c|c|c|c|c|c|c|c|c|c|c|c|c|c|c|}
\hline \multirow{2}{*}{$\begin{array}{c}\text { Feed } \\
\text { cost per } \\
100 \mathrm{lb} .\end{array}$} & \multicolumn{2}{|c|}{$\$ 0.15$} & \multicolumn{2}{|c|}{$\$ 0.17$} & \multicolumn{2}{|c|}{ \$0.19 } & \multicolumn{2}{|c|}{$\$ 0.21$} & \multicolumn{2}{|c|}{$\$ 0.23$} & \multicolumn{2}{|c|}{ \$0.25 } & \multicolumn{2}{|c|}{ so.27 } & \multicolumn{2}{|c|}{$\$ 0.29$} & \multicolumn{2}{|c|}{ S0.31 } \\
\hline & F & W & $\mathbf{F}$ & $w$ & F & w & $\mathbf{F}$ & $w$ & $\mathbf{F}$ & w & $F$ & $w$ & $\mathbf{F}$ & $\mathbf{w}$ & $\mathbf{F}$ & $\mathbf{w}$ & $F$ & w \\
\hline 3.00 & 392 & 120 & 67 & 153 & 722 & $|179|$ & 862 & 200 & 990 & $\mid 217$ &, 106 & 232 & 1,214 & 242 & 1,314 & 1202 & 1,407 & \\
\hline 25 & 280 & 96 & 455 & 132 & 611 & 160 & 750 & 183 & 878 & 202 & 994 & 217 & 4 & 231 & $\int 2$ & 245 & J & \\
\hline 50 & 176 & 83 & 1 & 112 & 507 & 142 & 647 & 166 & 774 & 187 & 891 & 204 & 1 & 222 & 1,098 & 231 & 1,22 & 2 \\
\hline 3.75 & 79 & 50 & 255 & 91 & 410 & $|123|$ & 550 & 150 & 678 & 171 & 794 & 190 & 4 & 210 & ,00Z & 219 & 14 & 2 \\
\hline 4 & & - & 164 & 70 & 320 & 105 & 460 & 133 & 582 & 156 & 4 & 176 & 812 & 192 & 912 & 207 &, 005 & 2 \\
\hline 4. & - & - & 9 & 50 & 235 & 87 & 375 & 116 & 502 & 141 & 619 & $|162|$ & 727 & 179 & 827 & 195 & 920 & 2 \\
\hline 4.50 & - & - & - & - & 155 & 68 & 295 & 100 & 422 & 126 & 539 & 148 & 647 & 166 & 747 & 182 & 840 & 1 \\
\hline 4.75 & - & - & - & - & 79 & 50 & 220 & 83 & 347 & 111 & 464 & 134 & 571 & 153 & 672 & 170 & 765 & \\
\hline 5.00 & - & - & - & - & 8 & 31 & 148 & 66 & 275 & 95 & 392 & 120 & 499 & 141 & 599 & 158 & 693 & 1 \\
\hline & - & - & - & - & - & - & 79 & 50 & 207 & 80 & 323 & 106 & 43 & $\mid 128$ & & 146 & 624 & \\
\hline 50 & 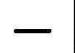 & - & - & - & 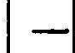 & 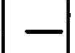 & 15 & 10 & 142 & 65 & 258 & 92 & 366 & 115 & 466 & 134 & 559 & 12 \\
\hline
\end{tabular}

${ }^{1} \mathrm{~F}=$ feed consumed to reach optimum weight; $W=$ weight of the animal for maximum profit. Pigs should not be raised under conditions designated by use of dashes.

to $\$ 5.50$ per 100 pounds. Overhead cost was not considered in the calculations. These costs vary greatly from farm to farm according to the management procedures followed. Obviously the smaller the overhead costs the larger the net profits. 


\section{THE TABLES}

The way to use tables 1 and 2 can be demonstrated by figuring a hypothetical situation in which feed price is $\$ 4$ per 100 pounds and the pigs are selling at $\$ 0.25$ per pound liveweight. For the Duroc barrows table 2 shows that under these conditions the optimum weight to which to carry the hogs would be 168 pounds. To attain that weight the pigs will have consumed 627 pounds of feed. The feed cost would be $\$ 25.08$ and the total selling price of each pig $\$ 42$, with a profit over the feed cost of $\$ 16.92$ per head.

\section{SUMMARY}

A study on the feeding of swine at the Lajas Experiment Substation shows the maximum weights that animals of the Duroc Jersey and Landrace $\mathrm{X}$ Duroc breeds should reach for best yield and maximum profit. Pigs considered in this study entered feeding trials which extended from weaning at 56 days to 182 days of age. Feed consumed and gain in weight of the pigs was recorded every 14 days. Reference tables included were worked out on this basis.

\section{RESUMEN}

El presente trabajo incluye información sobre un estudio llevado a cabo en la Subestación Experimental Agrícola en Lajas para determinar los pesos máximos a que se deben engordar los cerdos de las razas Duroc Jersey y Landrace $\mathrm{X}$ Duroc, de modo que la crianza de estos animales produzca los rendimientos mayores.

En los experimentos de alimentación se usaron cerdos desde la edad del destete (56 días) hasta que tuvieron 182 días de nacidos. Cada 14 días se anotaban las cantidades de alimentos consumidos, como también las ganancias en peso. Sobre esta base fué que se prepararon las tablas incluídas, de las cuales se puede deducir que bajo las condiciones de estos experimentos, el peso máximo a que se engordó un cerdo de la raza Duroc Jersey fué de 168 libras. Para llegar a este peso el animal consumió 625 libras de alimento con un valor de $\$ 25.08$. El precio de venta de este cerdo fué de $\$ 42.00$ según los precios que prevalecieron durante los experimentos y la ganancia alcanzó a $\$ 16.92$ por cabeza.

\section{LITERATURE CITED}

1. González Chapel, A., and Cabrera, J. I., Cross-breeding for swine production in Puerto Rico, J. Agr. Univ. P. R. 33 (4) 119-55, 1949. 